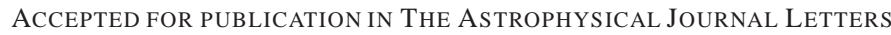

Preprint typeset using LTEX style emulateapj v. 11/10/09

\title{
LOCATION OF $\gamma$-RAY FLARE EMISSION IN THE JET OF THE BL LACERTAE OBJECT OJ287 MORE THAN 14 PC FROM THE CENTRAL ENGINE
}

\author{
Iván Agudo ${ }^{1}$, Svetlana G. Jorstad ${ }^{1,2}$, Alan P. Marscher ${ }^{1}$, VAleri M. LARionov ${ }^{2,3}$, José L. GómeZ ${ }^{4}$, AnNe LähteenmäKi ${ }^{5}$, \\ Mark Gurwell ${ }^{6}$, Paul S. Smith ${ }^{7}$, Helmut Wiesemeyer ${ }^{8}$, Clemens Thum $^{9}$, Jochen Heidt $^{10}$, Dmitriy A. Blinov ${ }^{2,3}$, \\ Francesca D. D'ArCangelo ${ }^{1,11}$, Vladimir A. Hagen-Thorn ${ }^{2,3}$, Daria A. Morozova ${ }^{2}$, Elina Nieppola ${ }^{5,12}$, Mar \\ ROCA-SOGORB ${ }^{4}$, GARY D. SCHMIDT ${ }^{13}$, BRIAN TAYLOR ${ }^{1,14}$, MERJA TORNIKOSKI ${ }^{5}$, IVAN S. TROITSKY ${ }^{2}$ \\ Accepted for publication in The Astrophysical Journal Letters
}

\begin{abstract}
We combine time-dependent multi-waveband flux and linear polarization observations with submilliarcsecond-scale polarimetric images at $\lambda=7 \mathrm{~mm}$ of the BL Lacertae-type blazar OJ287 to locate the $\gamma$-ray emission in prominent flares in the jet of the source $>14 \mathrm{pc}$ from the central engine. We demonstrate a highly significant correlation between the strongest $\gamma$-ray and millimeter-wave flares through Monte-Carlo simulations. The two reported $\gamma$-ray peaks occurred near the beginning of two major mm-wave outbursts, each of which is associated with a linear polarization maximum at millimeter wavelengths. Our Very Long Baseline Array observations indicate that the two mm-wave flares originated in the second of two features in the jet that are separated by $>14 \mathrm{pc}$. The simultaneity of the peak of the higher-amplitude $\gamma$-ray flare and the maximum in polarization of the second jet feature implies that the $\gamma$-ray and mm-wave flares are co-spatial and occur $>14 \mathrm{pc}$ from the central engine. We also associate two optical flares, accompanied by sharp polarization peaks, with the two $\gamma$-ray events. The multi-waveband behavior is most easily explained if the $\gamma$-rays arise from synchrotron self-Compton scattering of optical photons from the flares. We propose that flares are triggered by interaction of moving plasma blobs with a standing shock. The $\gamma$-ray and optical emission is quenched by inverse Compton losses as synchrotron photons from the newly shocked plasma cross the emission region. The mm-wave polarization is high at the onset of a flare, but decreases as the electrons emitting at these wavelengths penetrate less polarized regions.

Subject headings: Galaxies: active — galaxies: jets — BL Lacertae objects: individual (OJ287) — radio continuum: galaxies — gamma rays: general — polarization
\end{abstract}

\section{INTRODUCTION}

The information that $\gamma$-ray observations can provide on the physical properties of active galactic nuclei in general, and blazars in particular, depends on where such $\gamma$ ray emission originates, which is still under debate (e.g., Marscher \& Jorstad 2010). Variability studies of $\gamma$-ray and lower frequency emission from blazars provide important in-

\footnotetext{
${ }^{1}$ Institute for Astrophysical Research, Boston University, 725 Commonwealth Avenue, Boston, MA 02215, USA; iagudo@bu.edu

${ }^{2}$ Astronomical Institute, St. Petersburg State University, Universitetskij Pr. 28, Petrodvorets, 198504 St. Petersburg, Russia

${ }^{3}$ Isaac Newton Institute of Chile, St. Petersburg Branch, St. Petersburg, Russia

${ }^{4}$ Instituto de Astrofísica de Andalucía, CSIC, Apartado 3004, 18080, Granada, Spain

${ }^{5}$ Aalto University Metsähovi Radio Observatory, Metsähovintie 114, FIN-02540 Kylmälä, Finland

${ }^{6}$ Harvard-Smithsonian Center for Astrophysics, 60 Garden St., Cambridge, MA 02138, USA

${ }^{7}$ Steward Observatory, University of Arizona, Tucson, AZ 85721-0065, USA

${ }^{8}$ Instituto de Radio Astronomía Milimétrica, Avenida Divina Pastora, 7, Local 20, E-18012 Granada, Spain

${ }^{9}$ Institut de Radio Astronomie Millimétrique, 300 Rue de la Piscine, 38406 St. Martin d'Hères, France

${ }^{10}$ ZAH, Landessternwarte Heidelberg, Königstuhl, 69117 Heidelberg, Germany

${ }^{11}$ MIT Lincoln Laboratory, 244 Wood Street, Lexington, MA 02421, USA

${ }^{12}$ Finnish Centre for Astronomy with ESO (FINCA), University of Turku, Väisäläntie 20, FI-21500 Piikkiö, Finland

${ }_{13}$ National Science Foundation, 4201 Wilson Blvd., Arlington, VA 22230, USA

${ }^{14}$ Lowell Observatory, Flagstaff, AZ 86001, USA
}

sights into this problem by relating time scales of variability with physical sizes in the source. However, although $\gamma$-ray variability in blazars can occur on very short time-scales (of a few hours, e.g., Mattox et al. 1997; Foschini et al. 2010), this does not necessarily imply that high-energy flares take place at short distances $(\ll 1 \mathrm{pc})$ from the central engine (see Marscher \& Jorstad 2010). In fact, Lähteenmäki \& Valtaoja (2003) and Jorstad et al. (2001a b) provided evidence that $\gamma$ ray outbursts may arise in the mm-wave emitting regions $\gtrsim$ $1 \mathrm{pc}$ downstream of the central engine. The Large Area Telescope (LAT) onboard the Fermi Gamma-ray Space Telescope has sufficient sensitivity to test this by providing well-sampled light curves of blazars that allow detailed studies of the timing of $\gamma$-ray flares relative to those at other spectral ranges (e.g., Abdo et al. 2010d; Jorstad et al. 2010; Marscher et al.|2010).

The technique developed by Marscher et al. (2010) and Jorstad et al. (2010) uses ultra-high angular-resolution ( 0.15 milliarsecond) monitoring with very long baseline interferometry (VLBI) to resolve the innermost jet regions and monitor changes in jet structure. Observations with roughly monthly observations, supplemented by more concentrated campaigns, provide time sequences of total and polarized intensity images of the parsec-scale jet that can be related to variations of the flux and polarization at higher frequencies.

In this paper, we employ this technique to investigate the location of the flaring $\gamma$-ray emission in the BL Lacertae (BL Lac) object OJ287 $(z=0.306)$, a well studied and highly variable blazar at all available spectral ranges (e.g., Abdo et al. 2010a c). Throughout this paper we adopt the standard $\Lambda$ CDM cosmology with $H_{0}=71 \mathrm{~km} \mathrm{~s}^{-1} \mathrm{Mpc}^{-1}, \Omega_{M}=$ 
0.27 , and $\Omega_{\Lambda}=0.73$, so that 1 milliarcsecond (mas) corresponds to a projected distance of $4.48 \mathrm{pc}$, and a proper motion of $1 \mathrm{mas} / \mathrm{yr}$ corresponds to a superluminal speed of $19 c$.

\section{OBSERVATIONS}

Our polarimetric observations of OJ287 include (1) $7 \mathrm{~mm}$ (43 GHz) Very Long Baseline Array (VLBA) images (Fig.11), mostly from the Boston University blazar monitoring program 15 , (2) $3 \mathrm{~mm}(86 \mathrm{GHz})$ monitoring with the IRAM $30 \mathrm{~m}$ Telescope, and (3) optical ( $R$ and $V$ band) photo-polarimetric observations from several observatories (Figs. 2 and 3). The optical facilities include Calar Alto (2.2 m telescope, observations under the MAPCAT 6 program), Steward (2.3 and $1.54 \mathrm{~m}$ telescopes Petersburg State University ( $0.4 \mathrm{~m}$ telescope), and Crimean Astrophysical ( $0.7 \mathrm{~m}$ telescope) observatories. To these we add $R$-band polarimetric (and $V$-band photometric) data from Villforth et al. (2010). The total flux light curves analyzed here (see Fig. 2) are from the Fermi-LAT $\gamma$-ray $(0.1-$ $200 \mathrm{GeV})$ and Swift X-ray $(0.3-10 \mathrm{keV})$ and optical ( $V$-band) data available from the archives of these missions, by the Yale University SMARTS program 18, by the Submillimeter Array (SMA) at $1.3 \mathrm{~mm}(230 \mathrm{GHz})$ and $850 \mu \mathrm{m}(350 \mathrm{GHz})$, by the IRAM $30 \mathrm{~m}$ Telescope at $1.3 \mathrm{~mm}$, and by the Metsähovi Radio Observatory $14 \mathrm{~m}$ Telescope at $8 \mathrm{~mm}(37 \mathrm{GHz})$.

Our data analysis follows the procedures from previous studies: (1) VLBA: Jorstad et al. (2005); (2) optical polarimetric data: Jorstad et al. (2010); (3) IRAM data: Agudo et al. (2006, 2010); (4) SMA: Gurwell et al. (2007); (5) Metsähovi: Teräsranta et al. (1998); (6) Swift: Jorstad et al. (2010); and (7) Fermi LAT: Marscher et al. (2010). For the LAT data, we considered a $15^{\circ}$ radius centered on OJ287 and used the maximum-likelihood routine GTLIKE to model the $0.1-200 \mathrm{GeV}$ spectra from this object, CRATES J0856+2057, OJ 290, and 1FGL J0902.4+2050 as single power laws. We measured the flux in 7-day bins and fixed the slope of the photon spectrum of OJ287 at -2.4 , (as determined over the first 11 months of LAT observations by Abdo et al. 2010b).

\section{ANALYSIS OF OBSERVATIONAL RESULTS}

\section{1. $7 \mathrm{~mm}$ Jet Structure and Kinematics}

Fig. 1 reveals a radically different sub-milliarcsecondscale jet structure at $7 \mathrm{~mm}$ than found previously in OJ 287 . Jorstad et al. (2005) reported a bright core with a one-sided jet at a position angle $P A \approx-110^{\circ}$ in 1998-2001. Our new images show the innermost jet region oriented with $P A$ from $\sim-10^{\circ}$ to $\sim-40^{\circ}$ between 2008 and 2010, among the most extreme shifts in apparent jet direction yet observed (see e.g., Agudo et al. 2007). The change in orientation conflicts with the 12-yr period precession model presented by Tateyama \& Kingham (2004) that predicted the jet to be at $P A \approx-95^{\circ}$ in 2009 . A more detailed study of this issue and of the origin of jet wobbling in OJ287 will be presented elsewhere.

Jorstad et al. (2005) also reported a set of quasi-stationary features at average locations $\sim 0.1, \sim 0.3$, and $\sim 1$ mas from the innermost jet feature in OJ287. Fast superluminal features propagating downstream with speeds $\gtrsim 10 c$ were also

\footnotetext{
15 http://web.bu.edu/blazars/VLBAproject.html

16 http://www.iaa.es/ iagudo/research/MAPCAT

17 Data listing: http:// james.as.arizona.edu/ psmith/Fermi

18 Data listing: http://www.astro.yale.edu/smarts/glast
}

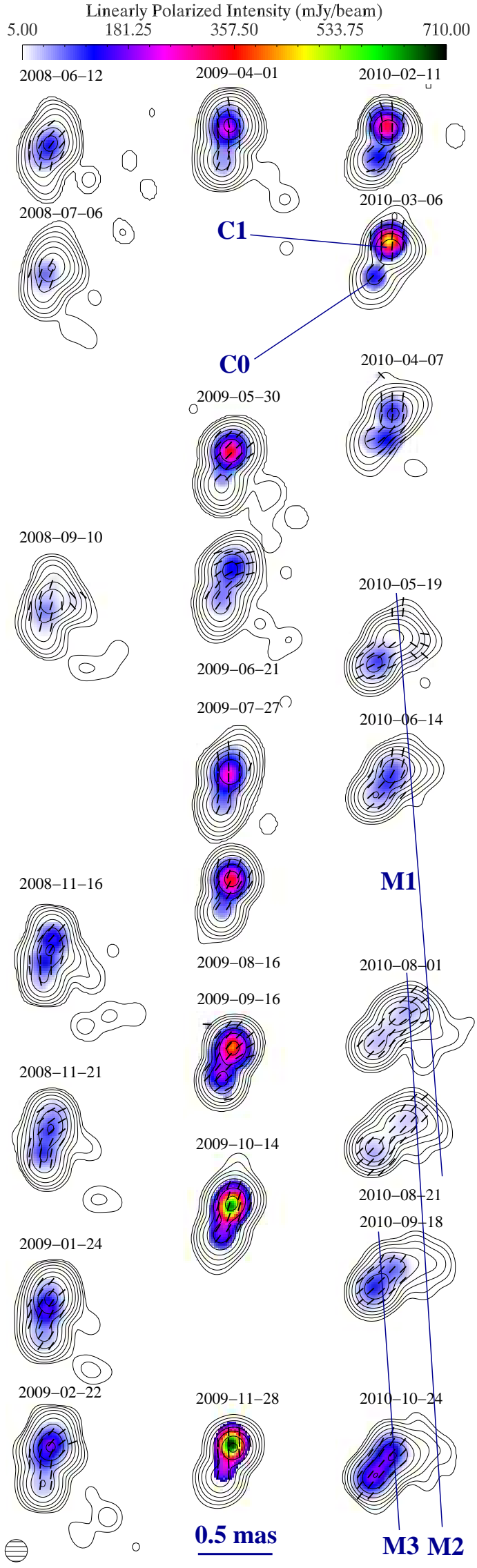

FIG. 1.- Sequence of $7 \mathrm{~mm}$ VLBA images of OJ287 in 2008-2010. Images are convolved with a FWHM $=0.15$ mas circular Gaussian beam. Contour levels represent $0.2,0.4,0.8,1.6,3.2,6.4,12.8,25.6,51.2,90.0 \%$ of the peak total intensity of $6.32 \mathrm{Jy} / \mathrm{beam}$. The color scale indicates linearly polarized intensity, whereas superimposed line segments represent the orientation of the polarization electric-vector position angle. 
observed to move across these quasi-stationary features. We model the brightness distribution of the source at $7 \mathrm{~mm}$ with a small number of circular Gaussian components. Our model fits include a bright quasi-stationary feature $(\mathrm{C} 1) \sim 0.2$ mas from the innermost jet region $(\mathrm{C} 0)$. The identification of $\mathrm{C} 0$ as the innermost jet feature is justified by the decreasing intensity westward of C1, and by the detection after 2010 March of superluminal motion of features M1 and M2 toward the westsouthwest of $\mathrm{C} 1$ with speeds of $10.8 c \pm 1.3 c$ and $6.7 c \pm 1.4 c)$ and possibly M3, which crossed C1 in 2010 Oct. (preliminary speed of $\gtrsim 10 c$ ).

\subsection{Flares in the C1 Jet Region at $1 \mathrm{~mm}$ and $7 \mathrm{~mm}$}

Before M1, M2, and M3 appeared in the jet, C0 and C1 typically contained $\gtrsim 90 \%$ of the $7 \mathrm{~mm}$ flux, and therefore governed the mm-wave evolution, of OJ287. In particular, the two most prominent $1 \mathrm{~mm}$ flares ever reported in OJ287 $\left(\mathrm{A}_{\mathrm{mm}}\right.$ and $\mathrm{B}_{\mathrm{mm}}$ as labeled in Fig. 2) took place in $\mathrm{C} 1$, as indicated by the correspondence of events in the $7 \mathrm{~mm}$ light curve of this component with those at other millimeter wavelengths. The multiple peaks of flare $B_{\mathrm{mm}}$ may be related to the passage of M1, M2, and M3 through C1, although we cannot verify this given the uncertainties in the trajectories of these knots. No moving features related to $\mathrm{A}_{\mathrm{mm}}$ are apparent in the images, although the polarization westward of $\mathrm{C} 1$ in the 2009-02-22 and 2009-06-21 images suggests that weak knots were present at those epochs.

From the angle of the jet axis to the line of sight in OJ287 (1.9-4.1; see Jorstad et al. 2005; Pushkarev et al. 2009), and the mean projected separation of $\mathrm{C} 1$ from $\mathrm{C} 0$ at the time of start of $A_{m m}$ and $B_{m m}(0.23 \pm 0.01$ mas $)$, we estimate that $C 1$ is located $>14 \mathrm{pc}$ downstream of $\mathrm{C} 0$, the innermost jet region detected in our images. The actual distance between the central engine in $\mathrm{OJ} 287$ and $\mathrm{C} 1$ must be even greater if $\mathrm{C} 0$ lies downstream of the acceleration and collimation zone of the jet (ACZ; Jorstad et al. 2007; Marscher et al. 2008, 2010).

\section{3. $\gamma$-Ray Flares}

The two most pronounced $\gamma$-ray flares take place during the initial rising phases of $\mathrm{A}_{\mathrm{mm}}$ and $\mathrm{B}_{\mathrm{mm}}$. This conforms with the pattern found in the 1990s by Lähteenmäki \& Valtaoja (2003). The two $\gamma$-ray flares, peaking on 2008 October $4\left(\mathrm{~A}_{\gamma}\right)$ and 2009 October $24\left(\mathrm{~B}_{\gamma}\right)$, reached $0.1-200 \mathrm{GeV}$ photon fluxes a factor of $\sim 2$ and $\sim 5$, respectively, higher than the quiescent $\gamma$-ray level $\left(\sim 1 \times 10^{-7} \mathrm{phot} / \mathrm{cm}^{2} / \mathrm{s}\right)$.

The discrete correlation function (DCF; Edelson \& Krolik 1988) between the $\gamma$-ray and $1 \mathrm{~mm}$ long-term light curve (Fig. 4 possesses a prominent peak at a time lag $\sim-80$ days ( $\gamma$-ray leading). We compute the significance of this result relative to stochastic variability through Monte Carlo simulations as in Chatterjee et al. (2008) and Max-Moerbeck et al. (2010). Our method follows Timmer \& Koenig (1995) for the simulation of $N=5000$ different pairs of $\gamma$-ray and $1 \mathrm{~mm}$ light curves characterized by the mean and standard deviation of the observed light curves, and by power-law shaped power spectral densities (PSD $\left.\propto 1 / f^{a}\right)$ with slopes in a grid $a_{\gamma}=\{1.0,1.5,2.0\}$ and $a_{1 \mathrm{~mm}}=\{1.0,1.5,2.0,2.5,3.0\}$. The range of $a_{\gamma}$ values includes the means found by Abdo et al. (2010c), $a_{\gamma}=1.5$ and 1.7 for quasars and BL Lacs, respectively. For $a_{1 \mathrm{~mm}}$, we choose values covering the range derived by Chatteriee et al. (2008) and Hufnagel \& Bregman (1992).

The results of our simulations show that the DCF peak at a lag of $\sim-80$ days is significant at $99.7 \%$ confidence in all

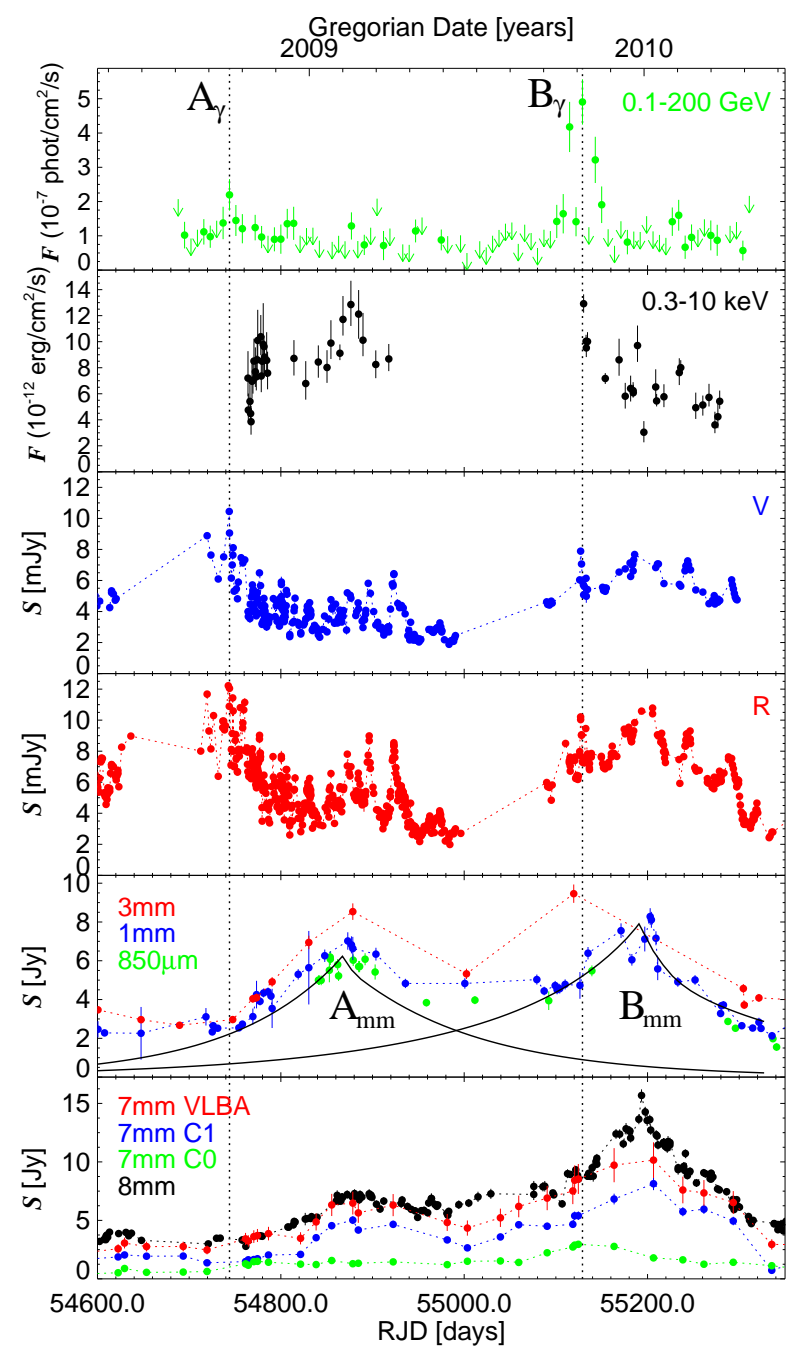

FIG. 2.- Light curves of OJ 287 from mm-wave to $\gamma$-ray frequencies. The vertical lines denote the times of peak $\gamma$-ray flux of $\mathrm{A}_{\gamma}$ and $\mathrm{B}_{\gamma}$. The solid lines in the next-to-last panel represent fits to the two major $1 \mathrm{~mm}$ flares by using the method of Chatteriee et al. (2008). RJD = Julian Date -2400000.0 .

of our simulations. This confirms the correlation between $\mathrm{B}_{\gamma}$ and $\mathrm{B}_{\mathrm{mm}}$, the most luminous $\gamma$-ray and $1 \mathrm{~mm}$ flares in our data. The correlation of the $\gamma$-ray and $8 \mathrm{~mm}$ light curves in Fig. 2] is of similarly high significance.

The optical light curves (especially $V$-band; Fig. 2) show two sharp flux increases at essentially zero time lag from $\mathrm{A}_{\gamma}$ and $\mathrm{B}_{\gamma}$. In contrast, the sparser time coverage of the $0.3-$ $10 \mathrm{keV}$ light curve does not allow us to make an unambiguous connection between the $\gamma$-ray and X-ray flares.

\subsection{Variability of Linear Polarization}

$\mathrm{C} 0$ and $\mathrm{C} 1$ dominate the evolution of the linear polarization $p$ and electric vector position angle $\chi$ at $7 \mathrm{~mm}$ in OJ287 (Fig. 3). However, whereas $p_{\mathrm{C} 0}$ never exceeds $10 \%, \mathrm{C} 1$ exhibits the two largest peaks in $p$ ever observed in OJ287 at $7 \mathrm{~mm}, p_{\mathrm{C} 1} \approx 14 \%$ on 2008 November 4 , and $p_{\mathrm{C} 1} \approx 22 \%$ on 2009 October 16 . The first maximum in $p_{\mathrm{C} 1}$ follows the peak of $\mathrm{A}_{\gamma}$ by one month, while the second more pronounced polarization event is already in progress when the $\gamma$-ray flux of flare $\mathrm{B}_{\gamma}$ rises to a level of $\sim 4 \times 10^{-7}$ phot $\mathrm{cm}^{-2} s^{-1}$. This coincidence of the strongest $\gamma$-ray outburst and exceptionally strong polarization in C1 identifies this feature $>14$ pc from the central engine as the site of the variable $\gamma$-ray emission. 


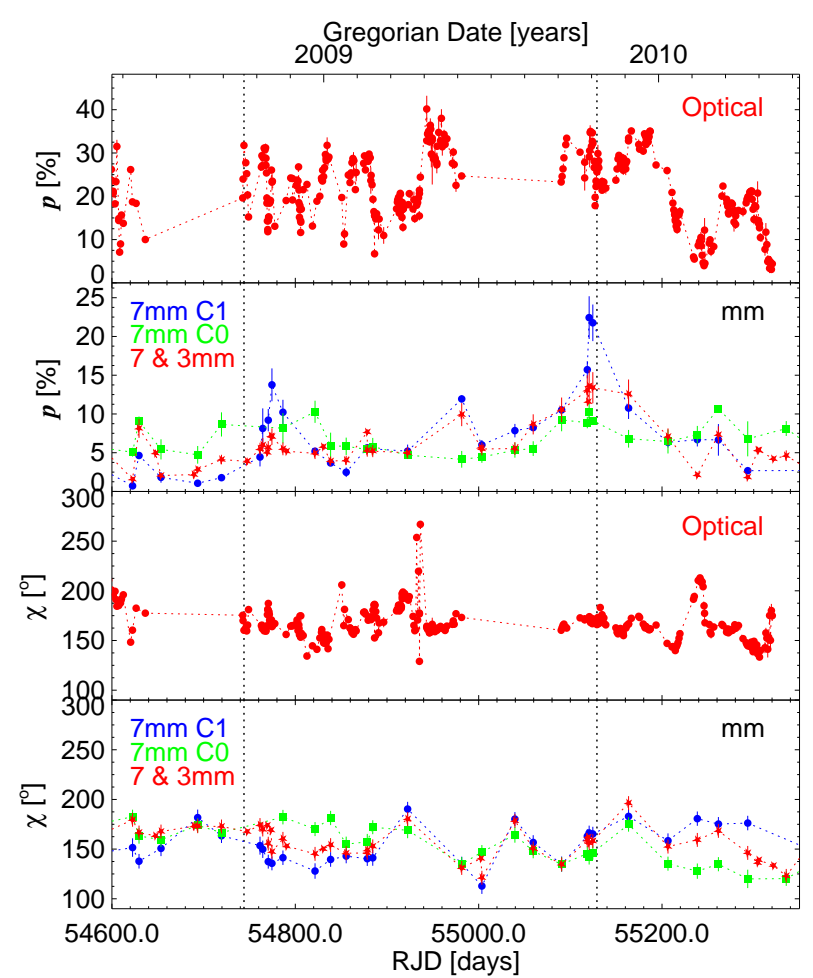

FIG. 3.- Optical and mm-wave linear polarization of OJ287 as a function of time. The optical data includes R-band and 5000-7000 $\AA$ observations. The similarity of the integrated linear polarization at $7 \mathrm{~mm}$ and $3 \mathrm{~mm}$ also allows us to combine them. Vertical lines are as in Fig.2

The optical polarization peaks at essentially the same time as $p_{\mathrm{C} 1}$ at $7 \mathrm{~mm}$ during flare $\mathrm{B}_{\gamma}$. During both $\mathrm{A}_{\gamma}$ and $\mathrm{B}_{\gamma}$, $p_{\text {opt }} \approx 35 \%$, which requires a well-ordered magnetic field. However, comparable optical polarization levels also occur at other times. The shorter time scale and larger amplitude of variability of optical polarization, as compared with those at millimeter wavelengths, is consistent with frequency dependence in the turbulence model of Marscher \& Jorstad (2010).

The optical and mm-wave linear polarization position angle is stable at $\chi \approx 160^{\circ}-170^{\circ}\left(-20^{\circ}\right.$ to $\left.-10^{\circ}\right)$ - similar to the structural position angle of the inner jet-both near $\mathrm{A}_{\gamma}$ and $\mathrm{B}_{\gamma}$ and throughout most of the monitoring period. The corresponding direction of the magnetic field is transverse to the direction between features $\mathrm{C} 0$ and $\mathrm{C} 1$. The stability of field direction is only greatly altered in the optical by sporadic short-term rotations of $\chi$ by up to $180^{\circ}$ when the polarization is relatively low (see Villforth et al. 2010), consistent with the behavior expected if the magnetic field becomes turbulent (Jones 1988; D’ Arcangelo et al. 2007).

\subsection{Low Probability of Chance Coincidences}

We note that the correlation peak in Fig. 4 is dominated by events $B_{\gamma}$ and $B_{m m}$. Despite the prominence of flare $A_{m m}$, the weakness of its $\gamma$-ray counterpart $\left(A_{\gamma}\right)$ prevents the pair of events from being apparent in the DCF at their time lag of $\sim-120$ days. However, there is statistical support for the hypothesis that flares $\mathrm{A}_{\gamma}$ and $\mathrm{A}_{\mathrm{mm}}$ are also physically related. The probability that a $\gamma$-ray outburst (approximated as instantaneous relative to the mm-wave flares) occurs by chance during a $\sim 120$-day rise time of a $1 \mathrm{~mm}$ flare peaking at $>5.5 \mathrm{Jy}$ is only $17 \%$. This probability is inferred from the duration

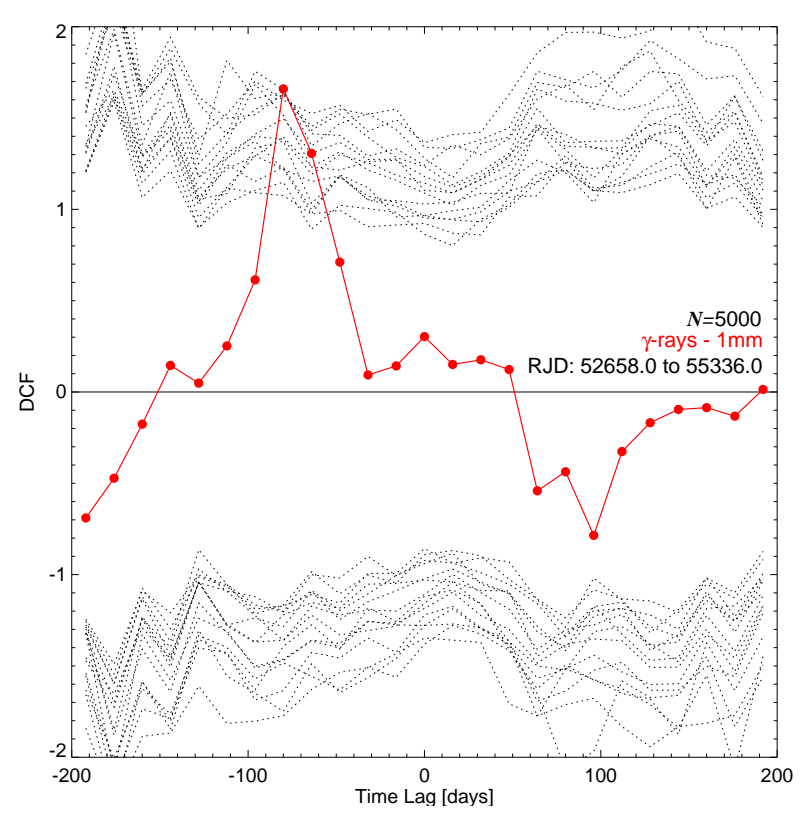

FIG. 4.- Discrete cross-correlation function between the $\gamma$-ray and $1 \mathrm{~mm}$ light curves of OJ287 (red points). The dotted curves at positive (negative) DCF values denote $99.7 \%$ confidence limits for correlation rather than stochastic variability for all combinations of $a_{\gamma}$ and $a_{1 \mathrm{~mm}}$ discussed in the text.

of the long-term $1 \mathrm{~mm}$ SMA light curve 19 and the number of such flares. Hence, the probability that two $\gamma$-ray flares at random times occur by chance during the rising phase of two mm-wave flares is $3 \%$, i.e., events $A_{m m}$ and $B_{m m}$ are associated with $\mathrm{A}_{\gamma}$ and $\mathrm{B}_{\gamma}$, respectively, at $97.0 \%$ confidence level. This rises to $99.2 \%$ if, instead of $A_{m m}$ and $B_{m m}$, the two high $p_{\mathrm{C} 1} \mathrm{~mm}$-wave peaks are considered.

\section{DISCUSSION AND CONCLUSIONS}

The two $0.1-200 \mathrm{GeV}$ flares in $\mathrm{OJ} 287$ allow us to assess the correspondence between $\gamma$-ray and lower-frequency variations. We find that two kinds of events at millimeter wavelengths are related to these $\gamma$-ray outbursts at high significance: (1) the early, rising phases of the two most luminous $1 \mathrm{~mm}$ flares ever detected in this blazar, $\left(\mathrm{A}_{\mathrm{mm}}\right.$ and $\left.\mathrm{B}_{\mathrm{mm}}\right)$; and (2) two sharp increases to unprecedented levels of linear polarization $(\sim 14 \%$ and $\sim 22 \%)$ in bright jet feature $\mathrm{C} 1>14 \mathrm{pc}$ from the central engine. These events also coincide with: (3) two sharp optical flares; (4) two peaks in optical polarization of $\sim 35 \%$; and (5) the similarity of optical and mm-wave polarization position angle both during and between the flares at $\chi \approx 160^{\circ}-170^{\circ}$.

The exceptionally high polarization of $\mathrm{C} 1$ during $\gamma$-ray flare $\mathrm{B}_{\gamma}$ provides extremely strong evidence that the event occurred in $\mathrm{C} 1$. This has two important implications. First, given the distance of $\mathrm{C} 1$ from the central engine, the $0.1-$ $200 \mathrm{GeV}$ flares must be produced by inverse Compton (IC) scattering rather than nuclear collisions. Second, the $\gamma$-ray IC emission arises from either the synchrotron self-Compton (SSC) process or IC scattering of infrared radiation from a hot, dusty torus of size $\sim 10 \mathrm{pc}$ (IC/dust; Błażejowski et al. 2004; Sokolov \& Marscher 2005). An SSC model is possible given the low ratio of $\gamma$-ray to synchrotron luminosity between $10^{14}$ and $10^{15} \mathrm{~Hz}(\approx 2)$ in OJ287, based on the fluxes we measure and a spectral index of -1.5 between $10^{14}$ and

19 http://sma1.sma.hawaii.edu/callist/callist.html 


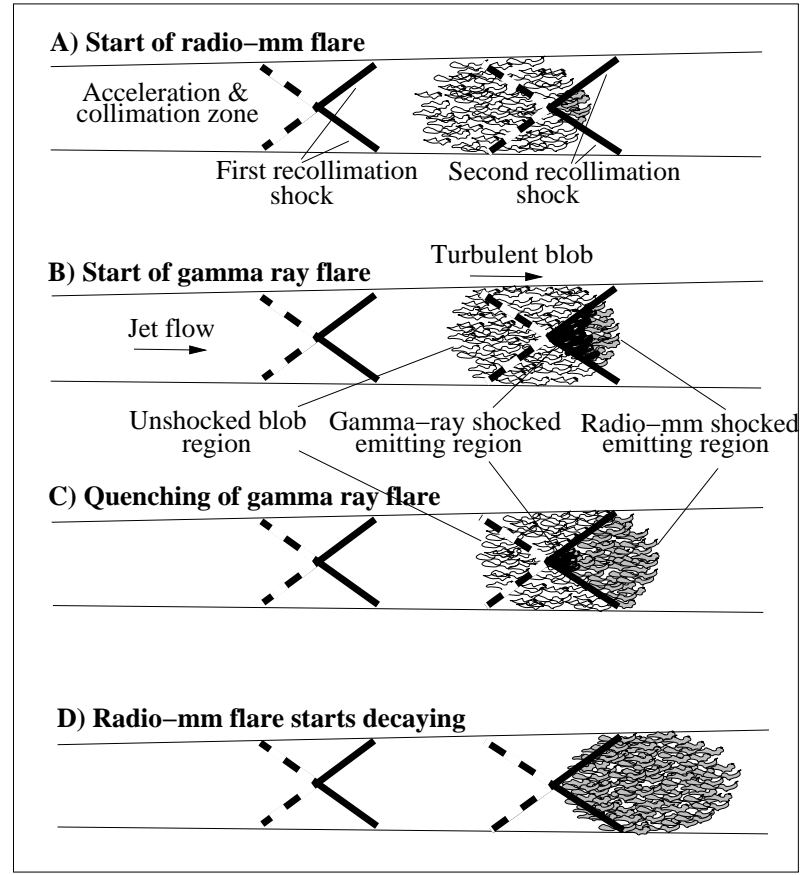

FIG. 5.- Sketch of the proposed model for the multi-wavelength flaring behavior of OJ 287.

$10^{15} \mathrm{~Hz}$ (Villforth et al. 2010). Inverse-Compton scattering of dust emission predicts that the optical and $\gamma$-ray emission should vary together, since the electron energies involved are similar (see, e.g., Marscher et al. 2010). This is the case during the $\gamma$-ray flares. The general data are therefore consistent with both the SSC and the IC/dust models.

The observed behavior agrees with a scenario in which the optical and $\gamma$-ray flares are produced in $\mathrm{C} 1$ by particle acceleration in a moving blob when it crosses a standing shock (Fig. 5) well beyond the ACZ. D'Arcangelo et al. (2007) have associated the innermost $\mathrm{mm}$-wave jet emission feature with a recollimation shock at the end of the ACZ. We identify $\mathrm{C} 0$ as such a feature, and $\mathrm{C} 1$ as a second re-collimation shock, as seen in hydrodynamical simulations (Gómez et al. 1997; Agudo et al. 2001) and often in VLBA images (Jorstad et al. 2001a, 2005). Jorstad et al. (2010) proposed a similar system of three conical shocks to explain the fine structure in the innermost $7 \mathrm{~mm}$ jet of 3C 454.3 in a feature that would be identified as the "core" at centimeter wavelengths. In OJ287, the higher flux of $\mathrm{C} 1$ relative to $\mathrm{C} 0$ could be caused by curvature in the jet increasing the Doppler beaming with distance from $\mathrm{C} 0$ so that it is maximized at $\mathrm{C} 1$.

A blob is either a shock or another disturbance that propagates down the jet with significantly higher relativistic electron density and magnetic field than in the ambient flow. The typical weakness of the observed polarization outside the high polarization peaks favors a turbulent plasma over a shock. Cawthorne (2006) shows that, in this case, the side of the conical shock nearest to the line of sight can be highly polarized with $\chi$ parallel to the jet axis. The remainder of the conical shock, farther from the line of sight, has much lower polarization. Because of light-travel delays, we first see the blob penetrate the near side, and therefore observe a major increase in polarization. As the outburst develops, more of the emission comes from the low-polarization far side, which decreases $p$ while the mm-wave flux density continues to increase. This is the pattern observed during both flares.

The mm-wave and optical flares start at essentially the same time as the magnetic field and electron energies near the leading edge of the blob become amplified as they pass the standing shock front. The mm-wave flux outburst continues as the relatively low-energy electrons fill the shocked region. The optical and $\gamma$-ray emission, produced by higher-energy electrons that cannot travel far before suffering radiative energy losses, is confined closer to the shock front where particles are accelerated (Marscher \& Gear 1985). If synchrotron losses were the sole factor, the optical and $\gamma$-ray flux would reach a plateau once the shocked blob plasma fills the entire layer behind the shock, declining only when the upstream side of the blob passes the shock front. However, if SSC emission dominates, the radiative losses will increase as synchrotron photons from the flare reach electrons that scatter them to high energies. The radiative energy losses then increase until the loss rate reaches a factor of $\left[1+\left(L_{\mathrm{SSC}} / L_{\mathrm{synch}}\right)\right](\approx 3$ in the case of OJ287) higher than the initial case of only synchrotron losses. This decreases the volume of emission as a function of time in the optical and $\gamma$-ray ranges. The timescale for this decrease is the light-travel time across the shock times $(1+z) \delta^{-1} \sim 20(\delta / 19)^{-1}(a / 0.054$ mas $)$ days, where $a$ is the cross-sectional angular size of the jet and the Doppler factor $(\delta)$ is that derived by Jorstad et al. (2005). This is similar to the time-scale of the $\gamma$-ray flux decline of flare $\mathrm{B}_{\gamma}(\sim 3-4$ weeks, see Fig. 2).

Quenching of the flares by increasing SSC energy losses operates only if the $\gamma$-rays are produced by the SSC process. Hence, the multi-frequency behavior of the flares is difficult to reproduce in the IC/dust model. Also, infrared emission from the dusty torus has not been detected thus far in BL Lacs such as OJ287, as far as the authors know. We thus favor the SSC mechanism.

The authors thank the referee for constructive comments. This research was funded by NASA grants NNX08AJ64G, NNX08AU02G, NNX08AV61G, and NNX08AV65G, NSF grant AST-0907893, and NRAO award GSSP07-0009 (Boston University); RFBR grant 09-02-00092 (St. Petersburg State University); MICIIN grants AYA200767627-C03-03 and AYA2010-14844, and CEIC (Andalucía) grant P09-FQM-4784 (IAA-CSIC); the Academy of Finland (Metsähovi); and NASA grants NNX08AW56S and NNX09AU10G (Steward Observatory). The VLBA is an instrument of the NRAO, a facility of the NSF operated under cooperative agreement by AUI. The PRISM camera at Lowell Observatory was developed by Janes et al., with funding from the NSF, Boston University, and Lowell Observatory. The Calar Alto Observatory is jointly operated by MPIA and IAA-CSIC. The IRAM $30 \mathrm{~m}$ Telescope is supported by INSU/CNRS (France), MPG (Germany), and IGN (Spain). The Submillimeter Array is a joint project between the SAO and the Academia Sinica.

\section{REFERENCES}


Abdo, A. A.,et al. 2010c, ApJ, 772, 520

Abdo, A. A., et al. 2010d, Nature, 463, 919

Agudo, I., et al. 2001, ApJ, 549, L183

Agudo, I., et al. 2006, A\&A, 456, 117

Agudo, I., et al. 2007, A\&A, 476, L17

Agudo, I., Thum, C., Wiesemeyer, H., \& Krichbaum, T. P. 2010, ApJS, 189, 1

Błażejowski, M., Siemiginowska, A., Sikora, M., Moderski, R., \& Bechtold, J. 2004, ApJ, 600, L27

Cawthorne, T. V. 2006, MNRAS, 367, 851

Chatterjee, R., et al. 2008, ApJ, 689, 79

D’Arcangelo, F. D., et al. 2007, ApJ, 659, L107

Edelson, R. A., \& Krolik, J. H. 1988, ApJ, 333, 646

Foschini, L., et al. 2010, MNRAS, 408, 448

Gómez et al. 1997, ApJ, 482, L33

Gurwell, M. A., Peck, A. B., Hostler, S. R., Darrah, M. R., \& Katz, C. A. 2007, in ASP Conf. Ser. 375, From Z-Machines to ALMA:

(Sub)millimeter Spectroscopy of Galaxies, ed. A. J. Baker et al. (San

Francisco, CA: ASP), 234

Hufnagel, B. R., \& Bregman, J. N., 1992, ApJ, 386, 473

Jones, T. W. 1988, ApJ, 332, 678
Jorstad, S. G., et al. 2001a, ApJS, 134, 181

Jorstad, S. G., et al. 2001b, ApJ, 556, 738

Jorstad, S. G., et al. 2005, AJ, 130, 1418

Jorstad, S. G., et al. 2007, AJ, 134, 799

Jorstad, S. G., et al. 2010, ApJ, 715, 362

Lähteenmäki, A., \& Valtaoja, E. 2003, ApJ, 590, 95

Marscher, A. P., \& Gear, W. K. 1985, ApJ, 298, 114

Marscher, A. P., et al. 2008, Nature, 452, 966

Marscher, A. P., \& Jorstad, S. G. 2010, in Fermi meets Jansky - AGN at Radio and Gamma-Rays, ed. T. Savolainen et al., 171

Marscher, A. P., et al. 2010, ApJ, 710, L126

Mattox, J. R., et al. 1997, ApJ, 476, 692

Max-Moerbeck, W., et al. 2010, in Fermi meets Jansky - AGN at Radio and Gamma-Rays, ed. T. Savolainen et al., 77

Pushkarev, A. B., Kovalev, Y. Y., Lister, M. L. \& Savolainen, T. 2009, A\&A, 507, L33

Sokolov, A., \& Marscher, A. P. 2005, ApJ, 629, 52

Tateyama, C. E., \& Kingham, K. A. 2004, ApJ, 608, 149

Teräsranta, H., et al. 1998, A\&AS, 132, 305

Timmer, J., \& Koenig, M. 1995, A\&A, 300, 707

Villforth, C., et al. 2010, MNRAS, 402, 2087 